\title{
Cell-type and transcription factor specific enrichment of transcriptional cofactor motifs in ENCODE ChIP-seq data
}

Chin Lui Goi ${ }^{1,3}$, Peter Little ${ }^{1,2,3^{*}}$, Chao Xie $2,3^{*}$

From Asia Pacific Bioinformatics Network (APBioNet) Twelfth International Conference on Bioinformatics (InCoB2013)

Taicang, China. 20-22 September 2013

\begin{abstract}
Background: Cell type and TF specific interactions between Transcription Factors (TFs) and cofactors are essential for transcriptional regulation through recruitment of general transcription machinery to gene promoter regions and their identification heavily reliant on protein interaction assays.

Results: Using TF targeted chromatin immunoprecipitation coupled with massively parallel sequencing (ChIP-seq) data from Encyclopedia of DNA Elements (ENCODE), we report cell type and TF specific TF-cofactor interactions captured in vivo through enrichments of non target cofactor binding site motifs within ChIP-seq peaks. We observe enrichments in both known and novel cofactor motifs.

Conclusions: Given the regulatory implications which TF and cofactor interactions have on a cell's phenotype, their identification is necessary but challenging. Here we present the findings to our analyses surrounding the investigation of TF-cofactor interactions encoded within TF ChIP-seq peaks. Novel cofactor binding site enrichments observed provides valuable insight into TF and cell type specific interactions driving TF interactions.
\end{abstract}

\section{Background}

Transcription Factors (TFs) are protein complexes responsible for the recruitment of basic transcription machinery to DNA [1]. At the molecular level, gene regulation is achieved via the binding of TFs to DNA but increases in complexity at a cellular level.

Distinct transcriptional programs made of interacting networks of TFs each regulating a subset of genes work cooperatively to generate the diversity of cells seen in multicellular organisms.

Mapping of TF interactions within such net- works are important for understanding of their control over gene expression and higher order functions such as cell

\footnotetext{
* Correspondence: Peter.little@nus.edu.sg; xiechao@bic.nus.edu.sg ${ }^{1}$ Department of Biochemistry, Yong Loo Lin School of Medicine, National University of Singapore, 8 Medical Drive, Singapore 117597, Singapore ${ }^{2}$ Life Sciences Institute, National University of Singapore, 28 Medical Drive, Singapore 117456, Singapore

Full list of author information is available at the end of the article
}

fate determination [2]. Although cell type specific expression of TFs have been identified, it is through combinatorial binding with partner TFs that the recruitment and formation of preinitiation complexes and RNA polymerases required for the transcription of cell type specific genes is achieved. Identification of such unique combinatory TF binding patterns occurring in a cell type specific manner is necessary for understanding of the unique transcriptional programmes which givejk rise to the repertoire of cell types seen in a multicellular organism $[1,3]$.

Cell type agnostic interactions do exist between TFs and are TF specific where master regulator TFs like the Signal Transducer and Activator of Transcription (STAT) bind with its cofactors to activate transcription of gene targets regardless of cell types $[4,5]$.

While existing methods of discovering TF-cofactor interactions require protein interaction assays, such as protein complex immunoprecipitation, or two hybrid screens 
which are low throughput, costly and non-indicative of in vivo conditions [6-10].

Specificity of TFs to their cognate binding sites have been well studied and with ChIP-sequencing (ChIP-seq) for chromatin immunoprecipitation (ChIP) coupled to ultra-high throughput massively parallel sequencing [11]. During ChIP-seq, DNA binding proteins are treated with a fixative agent, usually formaldehyde, and cross-linked to their bound DNA before it is extracted and the chromatin sheared to a size of $100-300$ bp $[12,13]$. The resulting protein(s) of interest in this case TFs are immunoenriched using an antibody precipitation targeted at the TF. Thereafter, the cross-links are reversed and the DNA purified and analysed by high-throughput DNA sequencing. Regions within the genome significantly mapped back onto are identified as potential protein-DNA interaction sites or peaks [11].

Sequenced regions include those bound by cofactors is largely due to the fixation step during ChIP where fixation not only occurs between the antibody targeted TFs and bound region but similarly with cofactors in a TFBSTF-Cofactor-TFBS manner [14]. As a result, protein-DNA interaction sites sequenced are not exclusive to the targeted TFs but also of their cofactors. Although, this has generally been viewed as noise and an artefact of the ChIP method impeding discovery of canonical TFBS motifs belonging to the targeted TF, documentation and support of enrichment of cofactor bound regions have been reported ranging from areas concerning peak calling techniques to genome-wide binding studies $[15,16]$.

Thus, identification and scanning of bound genomic regions by the ChIP targeted TFs and cofactors in vivo for transcription factor binding sites (TFBS) can be achieved and their co-occurrences used as a proxy for their interactions. While analysis of TF ChIP-seq peaks data is much more scalable for investigating far larger libraries of TFs.

Given the existence of cell type and TF specific TFcofactor interactions as well as the challenges in conventional methods of TF-cofactor discovery, we set out to explore in silico alternatives to analysing Cell type and TF specific TF-cofactor interactions from TFBS motif enrichments within ChIP-seq peaks. For cell type specific enrichments, we screened peaks for enrichments in non-canonical motifs, motifs with no known associations with the immuno-targeted TFs, across mutliple cell types (>10). Whereas for TF specific enrichments $>20 \%$ again in non-canonical motifs in $>3$ cell types targeting the same TF.

In our study we used human ChIP-seq data from The Encyclopedia of DNA Elements (ENCODE) Project [17]. In the June 2011 release by ENCODE, the Encode Transcription Factor Super Regulation Track integrates previously separate tracks containing ChIP-seq datasets from 81 experiments onto a single dataset which is mapped onto the latest human genome assembly (GRCh37/hg19). The release includes ChIP-seq experiments belonging to a variety of TFs carried out using different cell types.

In contrast to previous reports of cofactor sig-natures within TF ChIP-seq peak data [18] for our analysis, to our knowledge is the largest, spanning 81 ChIP-seq datasets after filtering.

For this study, we aim to identify TF-cofactor interaction networks through careful screening and analysis of transcription cofactor motifs captured by TF ChIP-seq as well as uncover nuances in their interaction specificities relating to cell types, and individual TFs.

\section{Results and discussion}

In the following, we begin with an overview of analyses conducted on the ChIP-seq dataset as well as report significant co-occurring TFBS motifs belonging to both validated and predicted cofactors. Of these, some exhibit Cell type as well as $T F$ specificity upon applying criteria specific filters.

\section{Overview}

Using ENCODE's recent release, a total of 425 ChIP-seq experiments studying $122 \mathrm{TFs}$ in 95 different cell cultures were considered initially for this study totaling $1,702,787$ unique ChIP-seq peaks.

We removed experiments investigating basic transcription machinery Polymerase I, II and III and non-canonical TF CTCF. Peaks belonging to high occupancy regions, that is being ubiquitous across ChIP-seq experiments regardless of conditions were also not considered. Finally, TFs investigated in only a single cell type as well as those without matched DNA binding site motifs were also removed. Also excluded from analysis were peaks showing extensive overlaps with peaks of other TF ChIP-seq experiments targeting different TFs $(67,246$ out of $1,702,787$ ). Accumulation of functionally unrelated DNA binding factors in regions known as 'High-Occupancy Target' (HOT) regions have been documented [19]. Nucleation at these sites has been shown mainly to be a result of protein-protein interactions [19-21] while proteinDNA interactions if any are still unclear hence, excluded.

The resulting dataset containing 1,022,885 peaks from 81 unique ChIP-seq experiments across 46 unique cell cultures of various tissue types was chosen. 28 unique TFs remained after curation with a total of 56 mapped canonical TFBS motif position weight matrix (PWMs).

Finally, we looked for cell type specific as well as TF specific co-occurrences and identified a total of 134 such motifs (Tables 1 and 2). Examples of the above will be discussed in the following. All identified co-occurring motifs and potential factors are provided in the supplementary (Additional Files 1 and 2). 
Table 1 Cell type specific co-occurring cofactor motifs.

\begin{tabular}{ccc}
\hline $\begin{array}{c}\text { Cell } \\
\text { type }\end{array}$ & Co-occurring motifs (Jaspar and Uniprobe Motif ID) & Total \\
\hline H1-hESC & MA0105.1, MA0145.1, MA0364.1, MA0375.1, MA0395.1, Zic1 secondary, Zic2 secondary, MA0154.1, MA0355.1, MA0364.1, Tcfap2b & 12 \\
& primary, Zic3 secondary & \\
HeLa-S3 & MA0145.1, MA0205.1, MA0375.1, MA0395.1, Zic2 secondary, Jundm2 secondary, MA0099.1, MA0272.1, MA0303.1 \\
HepG2 & MA0114.1, MA0017.1, MA0114.1 \\
K562 & MA0375.1, MA0395.1 & 3 \\
\hline
\end{tabular}

Listed in the table are motifs found consistently enriched within ChIP-seq peaks investigating a particular cell type independent of the TF targeted by the ChIP process.

\section{Proximally and distally located co-occurring motifs}

Peaks were later classified based on presence of canonical TFBS motifs belonging to the ChIP targeted TF $(322,085$ present and 700,800 absent) and a total of 75,955 non-canonical motifs were identified. Co-occurring motifs identified within peaks present and absent for the targeted TF's canonical motifs are thus classified as proximal and distal (Figure 1).

\section{Co-occurrence specificity of motifs}

To identify TF-cofactor networks operating proximally and distally through cis- and trans-acting elements with respect to the ChIP targeted TFs, we searched for enrichments in co-occurring TFBS motifs within ChIP-seq peaks. We based our search on three parameters namely: (1) motif abundance; the enrichment of the co-occurring motif in the ChIP-seq peaks, (2) motif ubiquity; the presence of the co-occurring motif across peaks from different TF ChIP-seq experiments and the (3) uniqueness or dis-similarity the targeted TF's canonical motif(s) using similarity scores with $p$-values $<0.05$. Potentially novel as well as known TF-cofactor pairs have been identified and selected examples will be discussed in the following. For the complete list of co-occurring motifs identified please refer to the supplementary tables provided (Additional files 1 and 2).

\section{Hepatocyte specific TF: HNF4 $\alpha$}

The most striking cell type specific enrichment observed belonged to the motif of Hepatocyte nuclear Factor 4 alpha $(H N F 4 \alpha)[22]$. The motif was found to be enriched both proximally and distally from ChIP targeted TF motifs found within the HepG2 cell lines regardless of the ChIP targeted TFs but not in other cell types (Figure 2).

Table 2 TF specific co-occurring cofactor motifs.

\begin{tabular}{|c|c|c|}
\hline $\begin{array}{l}\text { Target } \\
\text { TF }\end{array}$ & Co-occurring motifs (Jaspar and Uniprobe Motif ID) & Total \\
\hline c-Fos & MA0419.1, MA0316.1, MA0314.1, MA0315.1, MA0060.1 & 5 \\
\hline c-Jun & MA0419.1, MA0018.2, Atf1 primary, Jundm2 primary & 4 \\
\hline c-Myc & $\begin{array}{l}\text { Sp4 secondary, Zfp161 secondary, MA0324.1, Tcfap2e primary, MA0112.1, Plagl1 primary, MA0374.1, MA0014.1, Tcfap2a secondary, } \\
\text { Zic2 primary, Zic3 primary, Zic1 primary, MA0395.1 }\end{array}$ & 13 \\
\hline Egr-1 & Sp4 secondary, Zfp161 secondary, MA0324.1, MA0375.1, Tcfap2e primary, MA0374.1, MA0014.1, MA0268.1 & 8 \\
\hline GABP & $\begin{array}{l}\text { Sp4 secondary, Zfp161 secondary, MA0337.1, MA0324.1, MA0375.1, Tcfap2e primary, MA0048.1, MA0112.1, MA0374.1, MA0014.1, } \\
\text { MA0145.1, MA0138.2, Tcfap2a secondary, Zic2 primary, Zic3 primary, Zic1 primary, MA0145.1, Zic2 primary, Tcfap2b primary }\end{array}$ & 19 \\
\hline GATA-1 & $\begin{array}{l}\text { Mtf1 secondary, Tcfap2e secondary, MA0048.1, Srf secondary, Zfp105 primary, MA0402.1, MA0205.1, Sox13 secondary, Zic2 } \\
\text { secondary, Zic1 secondary, Tcfap2a secondary, MA0154.1, Zic3 secondary, MA0057.1 }\end{array}$ & 14 \\
\hline GATA-2 & Gata6 primary, Gata3 primary & 2 \\
\hline MafK & MA0419.1, Mtf1 secondary, Tcfap2e secondary, Srf secondary, Zfp105 primary, MA0099.2, MA0150.1, & 9 \\
\hline Max & Plagl1 primary & 1 \\
\hline NFKB & MA0364.1, Sox13 secondary, MA0154.1, Zic1 primary & 4 \\
\hline SP1 & $\begin{array}{l}\text { Sp4 secondary, Zfp161 secondary, MA0316.1, MA0315.1, Egr1 secondary, MA0112.1, Plagl1 primary, MA0374.1, MA0014.1, Sox13 } \\
\text { secondary, Tcfap2a secondary, 19 MA0314.1, MA0060.1, MA0057.1, MA0395.1, MA0060.1 }\end{array}$ & 19 \\
\hline STAT1 & Sox13 secondary & 1 \\
\hline STAT3 & MA0099.1, Jundm2 secondary, MA0272.1, MA0099.2, MA0303.1 & 5 \\
\hline USF-1 & Plagl1 primary, MA0314.1, MA0060.1 & 3 \\
\hline YY1 & Zic2 primary & 1 \\
\hline
\end{tabular}

Listed in the table are motifs found consistently enriched within ChIP-seq peaks investigating a particular TF independent of the cell type investigated. 


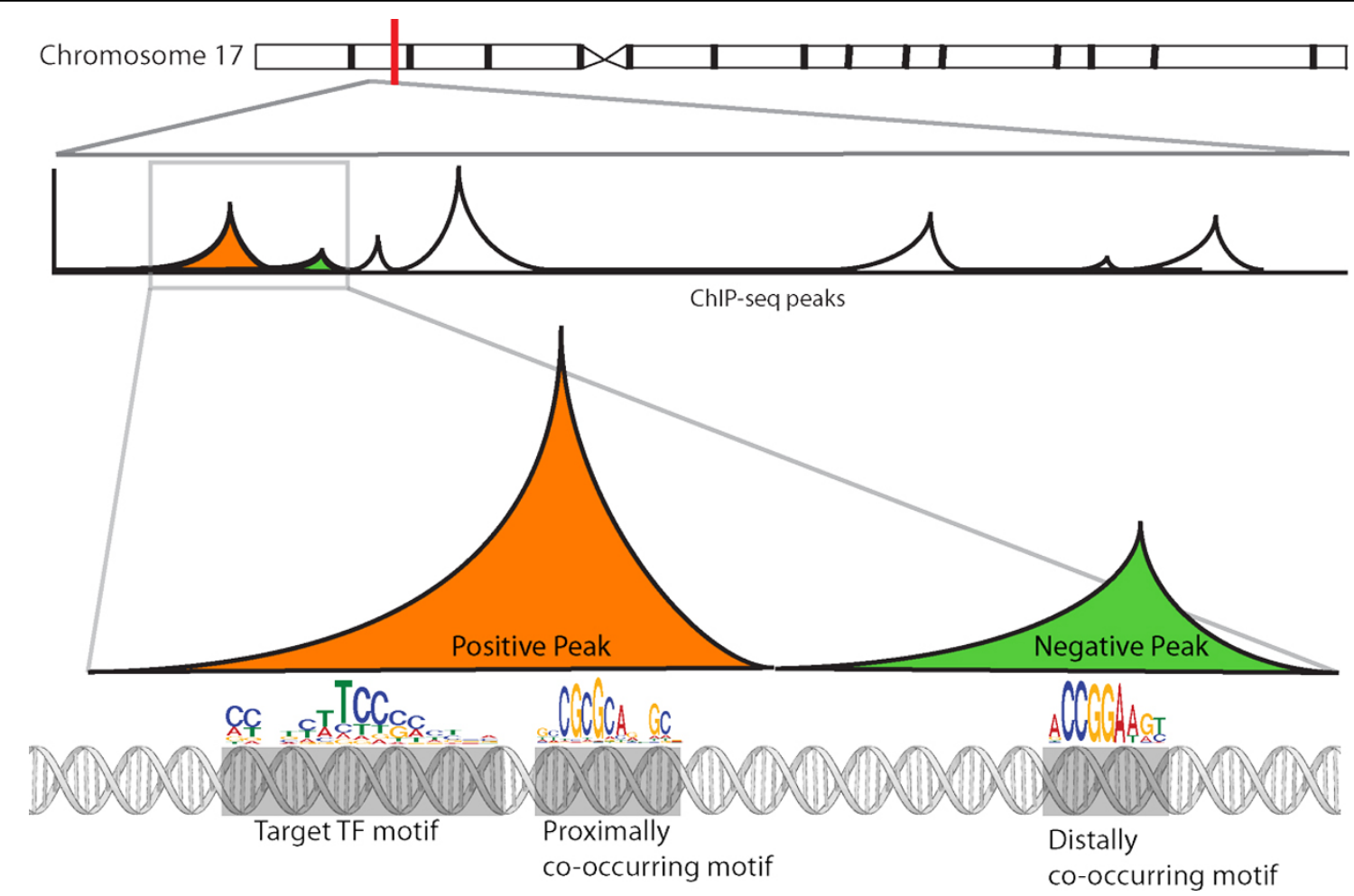

Figure 1 Diagrammatic representation of proximally and distally co-occurring motifs. In orange is a positive peak enriched with the target TF's motif as well as other proximally co-occurring motifs(s). In green, a negative peak absent for target TF's TFBS but enriched with cofactor motifs.

HNF4 $\alpha$ belongs to the superfamily of nuclear receptors known to be expressed endogenously in adult liver cell lines. Functionally, HNF4 $\alpha$ is a ligand-dependent transcription factor which is a master regulator for liver-specific gene expression and forms homodimers as well as heterodimers with other TFs via its AF-2 transactivation domain [23].

Co-occurring with HNF4 $\alpha$ motifs are the canonical motifs of 11 cofactor TFs (c-Jun, c-Myc, FOXA1, GABP, MafK, NRSF, RXRA, SP1, SRF, TBP, USF-1) both proximally and distally located peaks.

More cell type specific co-occuring TF motifs can be found in Additional file 3.

\section{TF specific co-occurring motifs}

As in the earlier section, we selected co-occurring motifs fulfilling the specific criteria of motif enrichment $(>20 \%)$ across $>3$ cell types targeting the same TF.

108 TF specific motifs were identified with the majority $(\sim 83 \%)$ proximal to target TFBS motifs. Of these are experimentally verified co-factors of the target TFs as well as those whose identity as a co-factor has not been experimentally verified (see Additional file 4).

In the following we will discuss briefly 4 examples of such motifs showing TF specific enrichment (2 belonging to known cofactors and 2 potentially novel cofactors).

\section{Examples of known associations}

\section{Signal Transducer and Activator of Transcription 3 and} Activator Protein-1

Upon applying the screening process, five TFBS motifs where found to be enriched proximally with canonical TFBS motifs of the Signal Transducer and Activator of Transcription Three (STAT3) (Jaspar motif ID: MA0144.1) regardless of cell type but not so in experiments targeting other TFs (Figure 3). Four out of five of these belonged to known cofactors and homologues of STAT3.

Three of the motifs identified were found to be canonical motifs of known STAT3 cofactor, Activator Protein 1 (AP-1) (Jaspar motif ID: MA0099.2) and its subunits c-Fos and c-Jun (Jaspar motif ID:MA0099.1 and Uniprobe motif ID: UP00103 secondary). Associations between STAT3 and AP-1 complexes are well characterised and their binding site motifs known to co-occur proximally together $[24,25]$. In addition, numerous assays con-ducted confirms their interactions both in vitro and in vivo [26-29].

AP-1 and STAT3 are known to be responsible for regulating the expression of genes mediating tissue repair and regeneration ubiquitously across cell types. The co-occurrences in the two's TFBS motifs in a cell type independent manner therefore is not surprising. 


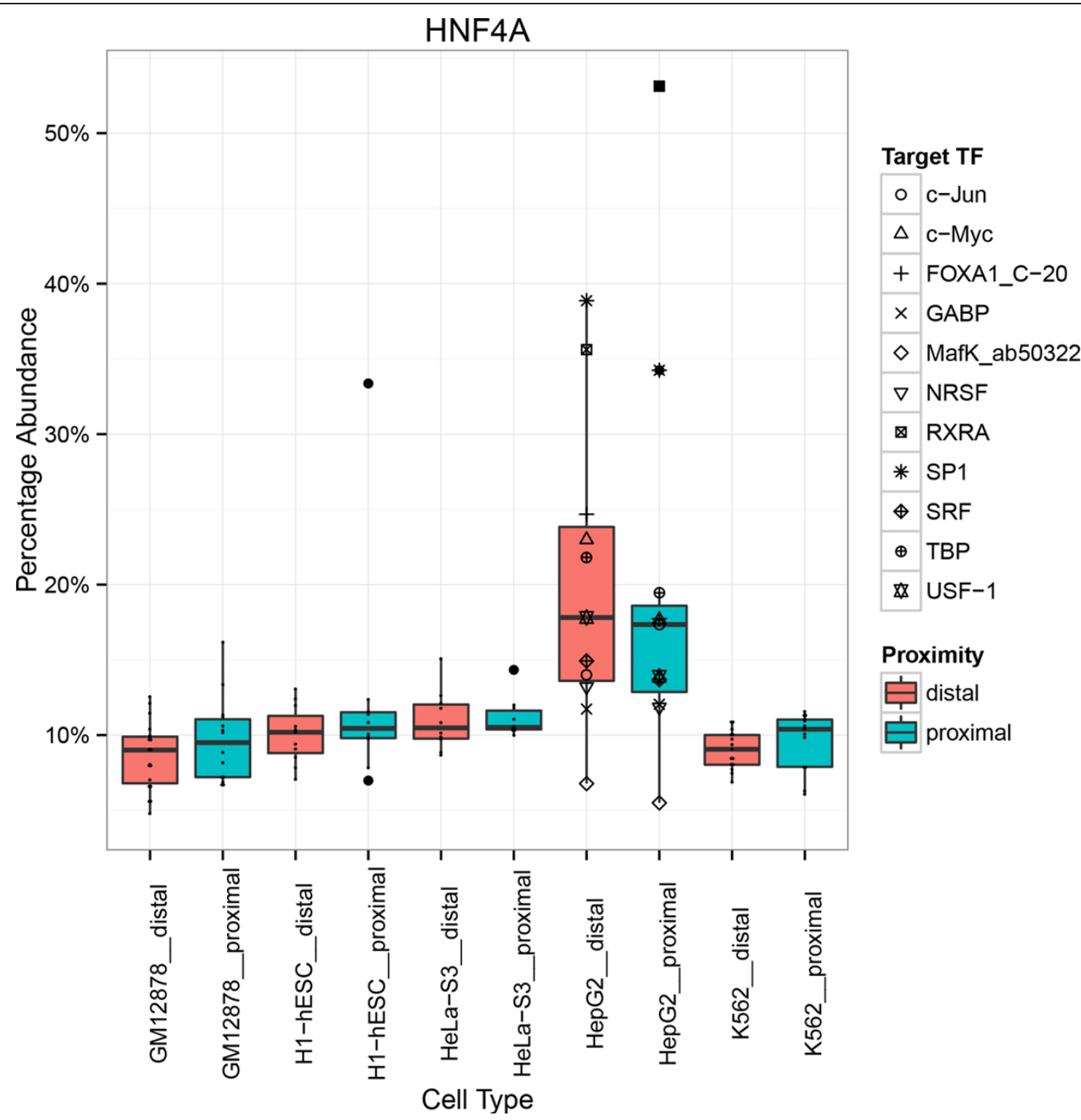

Figure 2 Distribution of cell type specific cofactor HNF4A binding site motif across cell types. Co-occurrence of transcription factor HNF4A's canonical motif in ChIP-seq peaks distal (pink) and proximal (green) with canonical motifs of ChIP targeted TFs across cell types. HNF4A binding site motif was found enriched in ChIP-seq peaks across experiments using HepG2 cell type. Dots represent individual ChIP-seq experiments.

Of the remaining two motifs, one is a Saccharomyces cerevisiae homologue of the AP-1 sub-unit c-Jun, GCN4 (Jaspar motif ID: MA0303.1) which binds to the AP-1 specific sequences ( $p$-value: $1.15405 \mathrm{e}-15)$ [30,31]. The other is a yeast TF responsible for regulating arginineresponsive genes [32-34].

Specificity Protein 1 (SP1) In a separate example of a TF specific co-occurrence of TFBS motifs, we observed the enrichment of 'CCAAT' family of TFs namely NFY, and the Saccharomyces cerevisiae homologues HAP3, HAP4 and HAP5 (Jaspar Motif ID: MA0060.1,
MA00314.1, MA00315.1 and MA00316.1) in proximal peaks of ChIP-seq experiments targeting SP1 across cell types (Figure 3). It has been documented that SP1 and NFY share large overlaps in promoter occupancies across numerous genes [35-37] as well as functional assays testing for co-operativity between the two [38].

The positive identification of AP-1 and its subunits' motifs in ChIP-seq peaks studying to STAT3 but not in peaks studying SP1 and vis versa acts simultaneously as a positive internal control as well as a negative internal control for this study. 


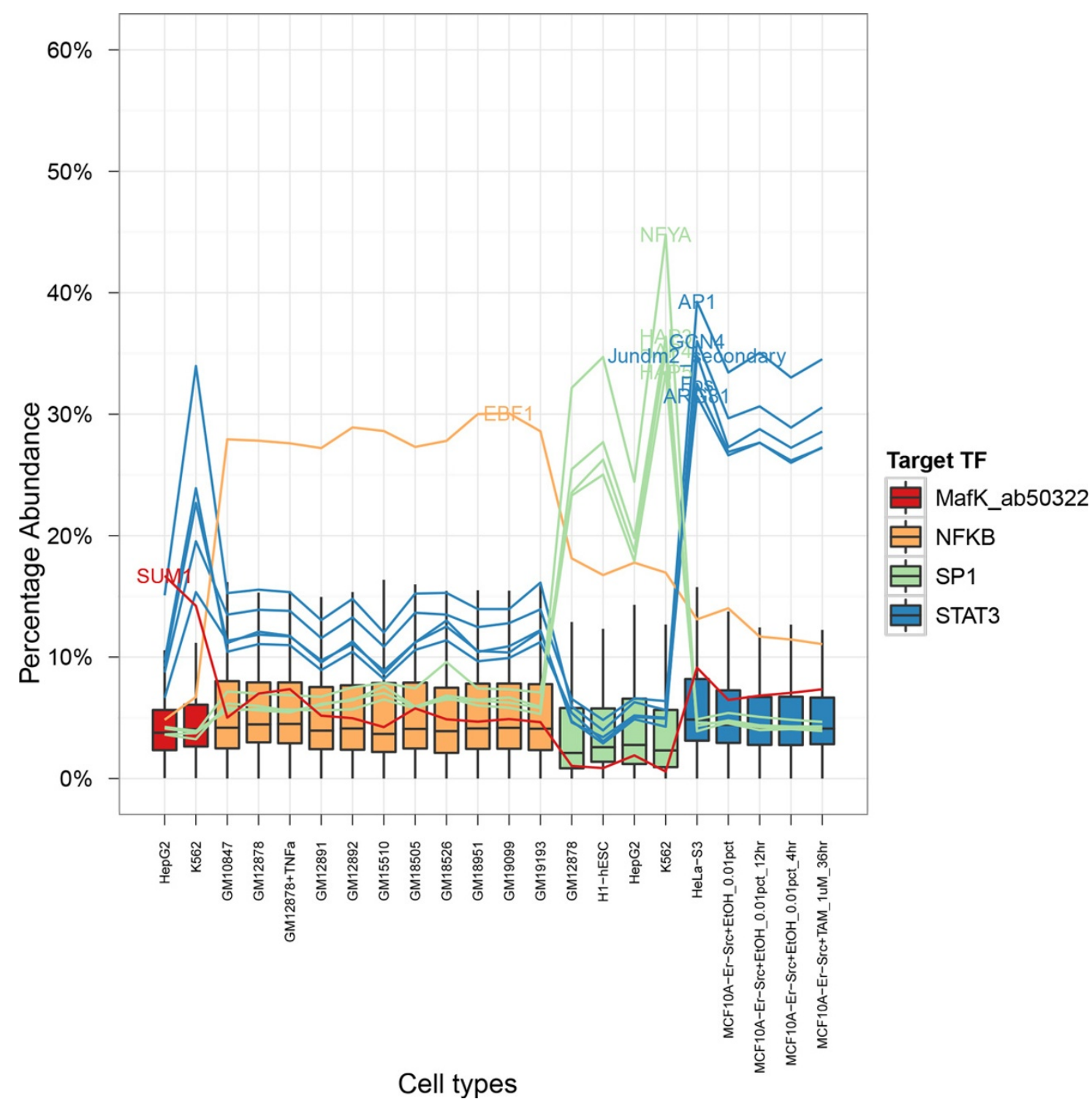

Figure 3 TF specific co-occurring cofactor motifs. TF specific co-occurring motifs identified upon applying the TF specificity screen. Box plot showing distribution of co-occurrence of motifs in ChIP-seq experiments investigating four TFs across various cell types. Overlaid on top of this is a line plot connecting TF specific motifs.

\section{Example novel cofactors $N F \kappa B$ and $E B F 1$}

TF specific motif co-occurrences identified in our analysis which have not been experimentally validated to our knowledge previously as a cofactors belong to EBF1 (Jaspar motif ID: MA0154.1). The co-occurrence was observed in the proximal peaks of ChIP-seq targeting NF $\kappa \mathrm{B}$ (Jaspar motif ID: MA0105.1) and the enrichment is fairly uniform across lymphocytes, embryonic stem cells, hepatocytes and human leukemia cells as shown in Figure 3.

EBF1 has been found to be important in the regulation of genes responsible for the normal progression of $\mathrm{B}$ cell development. Similarly, NF $\kappa \mathrm{B}$ too has been shown to be essential for proper B cell development [39,40]. Hence, the possibility of the two participating in some form of co-operative binding to regulate $\mathrm{B}$ cell development genes is high.

\section{Plagl1 and c-Myc}

The motif of Plagl1 (Uniprobe motif ID: UP00088) was found enriched within peaks from ChIP-seq experiments targeting c-Myc across cervix adenocarcinoma cells (HeLa), human leukemia cells (K562), hepatocytes (HepG2), human breast adenocarcinoma cells (Mcf-7), lymophocytes (GM12878) and promyelocytic cells (NB4).

Plagl1 and c-Myc are known regulators of the cell cycle and Plagl1 have been associated with inducing cell 
cycle arrest and apoptosis [41] while c-Myc involved in cell proliferation and apoptosis [42]. It is still unclear if the two TFs are true cofactors and will be a potential target for verification experimentally. In addition, the motif of Plagl1 was also identified in the peaks targeting SP1 and it is known that Plagl1 binds with SP1 response elements $[43,44]$. A summary figure showing the enrichment of the above mentioned pairing can be found in Figure 4.

\section{Conclusions}

Our analyses have uncovered enrichments of known and novel TF cofactors combinations occurring in cell-type and TF specific manner worth investigating. Through the examples discussed we've shown the retrieval of 3 verified cofactors including HNF4A in hepatocytes, STAT3 and AP-1, and NFY-A and SP1 as well as novel co-occurrences such as EBF1 and NFKB suggesting the possibility of the two being cofactors.

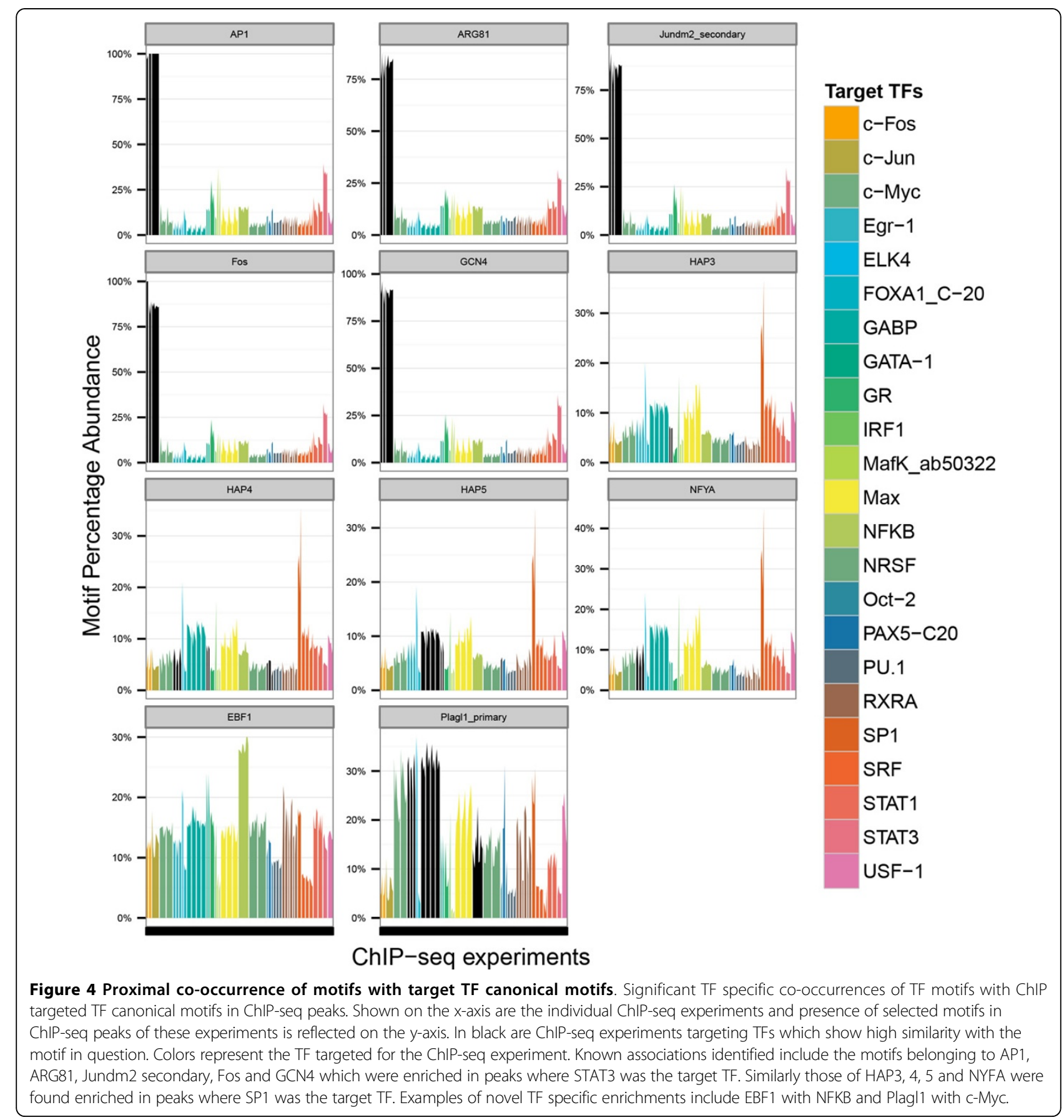


Thus, it is apparent following critical examination of enrichments in non-canonical TFBS motifs in ChIP-seq data that cofactor motifs signatures are indeed detectable and retrievable through rigorous screening as described in our study.

In conclusion, we have shown through careful examination of motif enrichment in ChIP-seq data that not only are global cofactors of TFs be identified but also criteria specific binding partners. This could potentially be used for quick identification of potential cofactors of newly characterised TFs not only in humans but also other model organisms.

Such analyses as described in our study will prove be invaluable as more TFs are interrogated using ChIP-seq as the cost of next generation sequencing continues to become more affordable.

\section{Methods}

To identify potential cofactor TFBS motifs from ChIP-seq data, we began with the collection of TF ChIP-seq experimental data as well as a representative list of known TFBS motifs. Next, we applied a series of procedures to process and screen for significant motifs exhibiting any of the two qualities: Cell type and TF specificity.

\section{Preparation and integration of data TF ChIP-seq data}

In our analysis, we used TF ChIP-seq experimental data retrieved from the Integrated Transcription Factor Track in the Data Coordination Center of the ENCODE project $[45,46]$. The Integrated Transcription Factor Track was downloaded as a flat data matrix consisting of the genomic coordinates of ChIP-seq peaks corresponding to cell type of the experiment and TF investigated. Data from a total of 425 ChIP-seq experiments were retrieved, featuring 122 TFs targeted using a total of 148 TF specific antibodies in 95 different cell cultures be-longing to 71 unique cell types some treated with biological or non-biological agents.

All 1,702,787 peaks were mapped to their appropriate DNA sequences belonging to the latest human genome assembly (GRCh37/hg19) [45,46] using the getSeq() function from the BSgenome.Hsapiens.UCSC.hg19 package in $\mathrm{R}$ [47].

\section{TFBS motif Position Weight Matrices (PWMs)}

Identification of co-occurring TFBS motifs using motif finding algorithms requires consensus Position Weight Matrices (PWMs) which summarise DNA profiles of DNA sites bound by the DNA binding domain (DBDs) of a TF. We retrieved curated PWMs from two leading open-access TFBS motif databases: JASPAR CORE 2009 and UniPROBE Mouse [48,49]. Entries from the two databases show little overlap, representative of all known TFBS motifs used to search TFBS motifs in ChIP-seq peaks.

\section{Matching target TFs to PWMs}

A curated list of Tar-get TF canonical TFBS PWM(s) was retrieved from Ensembl [50].

\section{A high level procedure for selecting ChIP-seq peaks and} scanning for enriched cofactor TFBS motifs

We begin by examining data from each ChIP-seq experiment based on the following: the nature of the targeted TF, the number of experiments targeting the same TF, peak density of genomic regions and associated peaks, and the availability of target TF TFBS motif PWM(s).

Thereafter, we searched for proximally and dis-tally located co-occurring motifs by scanning for motifs in peaks positive for the target TFBS motif and peaks negative for the target TFBS motif. Motifs identified in the former represent motifs found in close proximity with the target TFBS while the motifs identified in the later represent motifs located distally from the target TFBS.

Finally, we determined significant and non-ubiquitous co-occurrences and screened them for: Cell type and TF specificity.

\section{Data curation}

\section{Nature of ChIP-seq targeted transcription factors}

For meaningful analysis of TF-cofactor interactions, ChIP-seq experiments targeting general transcription machinery such as Polymerase II, III and the TATAbinding protein (TBP) were not considered for analysis. Similarly, the non-canonical TF such as CTCF were also removed.

Justifications for considering CTCF as a non-canonical TF Initially considered as a canonical TF, CCCTC-binding Factor (CTCF) shows similar genomic distributions with TFs such as STAT1 and NRSF [51]. However, CTCF has also been shown to exhibit additional non-canonical qualities acting as a transcriptional insulator as well as binding with multiple divergent DNA motifs [52]. In addition, CTCF exhibits large deviations in its genome-wide distribution from Transcription Start Sites (TSS) when compared to general transcription machinery and only $\approx 20 \%$ of its binding sites show promoter-proximal localisation [53]. Considering CTCF's non-canonical TF qualities, experiments targeting CTCF were therefore not included for analysis.

\section{Number of cell type specific experiments targeting the} same TF

'Orphan' ChIP-seq experiments without 'sister' experiments investigating the same TF but in different cell types were not selected for further analysis as we were unable to ascertain occurrence specificity of the co-occurring motifs.

\section{Peak occupancy in mapped regions}

HOT and COLD regions Individual peaks from each ChIP-seq experiment were curated based on the TF occupancy of the regions they are found in. Regions 
observing significant overlaps in peaks from multiple TFs (ChIP-seq experiments), henceforth referred to as High-Occupancy-Target (HOT) regions, are known to se-quester DNA binding factors but yet not much is known about their formation. As we were unable to determine if the motifs in the DNA sequences coimmunoprecipitated were truly bound by a cofactor or simply by another factors in the larger protein aggregates we chose not to include the peaks coming from these HOT regions into our analysis.

The arbitrary cutoff set to delimit such HOT regions requires the overlapping of peaks from more than 50\% of all ChIP-seq experiments investigated. Peaks found in these HOT regions will be henceforth referred to as "HOT peaks" and the rest as "COLD peaks" for nomenclatural reasons. Availability of Target TF TFBS motif PWM(s) Experiments targeting TFs without any matched TFBS motif PWM(s) from the curated list of TF PWMs retrieved from Ensembl earlier were removed from analysis.

TFs which were matched to multiple canonical motifs were also observed due to multiple DBDs or have DBDs with alternative conformational states.

\section{Proximally and distally co-occurring motifs}

As shown in Figure 1, there exists proximally and distally located co-occurring motifs captured by ChIP-seq. Using canonical TFBS motif(s) for each TF, we scanned the corresponding ChIP-seq peaks for their presence and segregated the peaks into two; positive or negative. Next, we scanned both positive and negative peaks for TFBS motifs from our com-piled library of TFBS motifs.

Enrichment of TFBS motifs excluding that of the target TF's in positive peaks were considered to be proximal co-occurrences where the both target TF motif and enriched motif share the same ChIP-seq peak. Motifs enriched in peaks negative for the target TF's motif(s) were grouped as distally co-occurring. See Figure 1 for a diagrammatic representation of positive and negative peaks as well as proximally and distally located co-occurring motifs.

\section{Motif enrichment abundance}

Abundance scores for each of the identified co-occurring motifs were assigned based on the motif's presence across the ChIP-seq peaks investigated regardless of its enrichment within each peak.

\section{Ubiquity of motifs across experiments}

Some motifs were observed to co-occur in ChIP-seq peaks both abundantly within a ChIP-seq experiment as well as ubiquitously across ChIP-seq experiments regardless of the cell type or the targeted TF. Such non-specific motifs were discarded from further analysis as we proceeded to screen for various criteria specific co-occurrences of TFBS motifs in the second part of our analyse.
This was achieved using two arbitrary thresholds, such that the selected motifs would not be co-occurring abundantly within an experiment (95th percentile) as well as be not too ubiquitous across ChIP-seq experiments (proximal: $<20 \%$ and distal: $<10 \%$ ). The second threshold was chosen based upon the relative abundance of co-occurring motifs across experiments after applying the first threshold.

Cell type specific Motifs must co-occur in ChIP-seq peaks of experiments investigating the same cell type but across TFs $(>3)$ and enriched with abundances above $15 \%$. Motifs must co-occur in at least ten individual ChIP-seq experiments investigating the same cell type but different TF.

TF specific Motifs must co-occur with the same target TF motif in at least three or more 'sister' experiments investigating other cell types. The motif must also have enrichment abundances above $20 \%$ in all experiments.

\section{Identifying TFBS motifs in DNA sequence data from ChIP-seq}

To scan for the relative occurrence of TFBS motif PWMs within ChIP-seq peaks, we used the TFBS motif identification software, FIMO, for Find Individual Motif Occurrences. FIMO is found as a part of the MEME suite of motif analysis algorithms and requires PWM(s) of the queried TFBS motif for scanning and identifying TFBS motifs in the sequences provided. When comparing two motifs, FIMO assigns a log-likelihood ratio score to each sequence position matched and the scores are converted into a $p$-value representing the probability of a true match. A corresponding $\mathrm{q}$-value is generated using a bootstrap method to determine the mini-mal false discovery rate at which the $p$-value will be deemed significant [54].

\section{Evaluating motif similarity}

The varying degrees of similarity between motifs found in the compiled list of motifs were determined using the motif similarity comparison software, TOMTOM. TOMTOM creates ungapped alignments between queried motifs against a database of PWMs and determines a $p$-value describing the significance of the match against the rest of the motifs in the database. [55]. For this experiment all com-piled motifs were used as the reference database.

Motifs with $p$-values scores less than 0.05 , when compared with the target TF's motif(s) were considered to be false positives.

\section{Additional material}

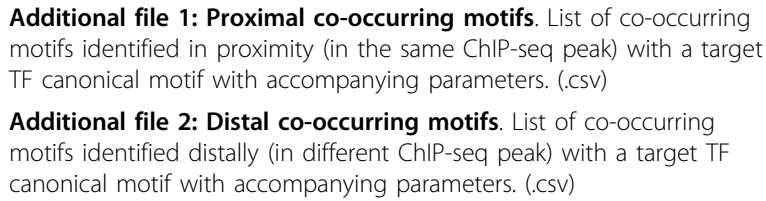

Additional file 1: Proximal co-occurring motifs. List of co-occurring motifs identified in proximity (in the same ChIP-seq peak) with a target TF canonical motif with accompanying parameters. (.csv)

Additional file 2: Distal co-occurring motifs. List of co-occurring motifs identified distally (in different ChIP-seq peak) with a target TF canonical motif with accompanying parameters. (.csv) 
Additional file 3: Cell type specific motifs. List of cell type specific cooccurring motifs. (.csv)

Additional file 4: TF specific motifs. List of TF specific co-occurring motifs. (.csv)

\section{Competing interests}

The authors declare that they have no competing interests.

\section{Authors' contributions}

Experimental design by $C X$ and $P L$ and data analysis by $G C L$ with assistance from CX. Manuscript written by GCL with input from CX and PL. All authors read and approved the final manuscript.

\section{Acknowledgements}

We thank Lim Kwan Siong, Mark De Silva for technical assistance. We also thank students and staff of Bioinformatics Center, Biochemistry, National University of Singapore for their support throughout the duration of the project.

\section{Declaration}

Publication of this article was supported by a grant to PL from the National University of Singapore (Grant R-069-000-047).

This article has been published as part of BMC Genomics Volume 14 Supplement 5, 2013: Twelfth International Conference on Bioinformatics (InCoB2013): Computational biology. The full contents of the supplement are available online at http://www.biomedcentral.com/bmcgenomics/ supplements/14/S5.

\section{Authors' details}

'Department of Biochemistry, Yong Loo Lin School of Medicine, National University of Singapore, 8 Medical Drive, Singapore 117597, Singapore. ${ }^{2}$ Life Sciences Institute, National University of Singapore, 28 Medical Drive, Singapore 117456, Singapore. ${ }^{3}$ Singapore Centre on Environmental Life Sciences Engineering (SCELSE) Nanyang Technological University 60 Nanyang Drive, SBS-01N-27 Singapore 637551, Singapore.

Published: 16 October 2013

\section{References}

1. MacQuarrie KL, Fong AP, Morse RH, Tapscott SJ: Genome-wide transcription factor binding: beyond direct target regulation. Trends Genet 2011, 27(4):141-148.

2. Sridharan R, Tchieu J, Mason MJ, Yachechko R, Kuoy E, Horvath S, Zhou Q, Plath K: Role of the murine reprogramming factors in the induction of pluripo-tency. Cell 2009, 136:364-377.

3. Vaquerizas JM, Kummerfeld SK, Teichmann SA, Luscombe NM: A census of human transcription factors: function, expression and evolution. Nat Rev Genet 2009, 10:252-263

4. Dang CV, O'Donnell KA, Zeller KI, Nguyen T, Osthus RC, Li F: The c-Myc target gene network. Semin Cancer Biol 2006, 16:253-264.

5. Robertson G, Hirst M, Bainbridge M, Bilenky M, Zhao Y, Zeng T, Euskirchen G, Bernier B, Varhol R, Delaney A, Thiessen N, Griffith OL, He A, Marra M, Snyder M, Jones S: Genome-wide profiles of STAT1 DNA association using chromatin immunoprecipitation and massively parallel sequencing. Nat Methods 2007, 4:651-657.

6. Yu H, Braun P, Yildirim MA, Lemmens I, Venkatesan K, Sahalie J, HirozaneKishikawa T, Gebreab F, Li N, Simo-nis N, Hao T, Rual JF, Dricot A, Vazquez A, Murray RR, Simon C, Tardivo L, Tam S, Svrzikapa N, Fan C, de Smet AS, Motyl A, Hudson ME, Park J, Xin X, Cusick ME, Moore T, Boone C, Snyder M, Roth FP, Barabasi AL, Tavernier J, Hill DE, Vidal M: High-quality binary protein interaction map of the yeast interactome network. Science 2008, 322(5898):104-110.

7. Park D, Lee S, Bolser D, Schroeder M, Lappe M, Oh D, Bhak J: Comparative interactomics analysis of protein family interaction networks using PSIMAP (protein structural interactome map). Bioinformatics 2005, 21(15):3234-3240.

8. Fields S, Song O: A novel genetic system to detect protein-protein interactions. Nature 1989, 340(6230):245-246.
9. Parrish $J R$, Gulyas KD, Finley RL: Yeast two-hybrid contributions to interactome mapping. Curr Opin Biotechnol 2006, 17(4):387-393.

10. Koegl M, Uetz P: Improving yeast two-hybrid screening systems. Brief Funct Genomic Proteomic 2007, 6:302-312.

11. Pepke S, Wold B, Mortazavi A: Computation for ChIP-seq and RNA-seq studies. Nat Methods 2009, 6:22-32.

12. Ren B, Robert F, Wyrick JJ, Aparicio O, Jennings EG, Simon I, Zeitlinger J, Schreiber J, Hannett N, Kanin E, Volkert TL, Wilson CJ, Bell SP, Young RA: Genome-wide location and function of DNA binding proteins. Science 2000, 290(5500):2306-2309.

13. Iyer VR, Horak CE, Scafe CS, Botstein D, Snyder M, Brown PO: Genomic binding sites of the yeast cell-cycle transcription factors SBF and MBF. Nature 2001, 409(6819):533-538.

14. Farnham PJ: Insights from genomic profiling of transcription factors. Nat Rev Genet 2009, 10:605-616.

15. Valouev A, Johnson DS, Sundquist A, Medina C, Anton E, Batzoglou S, Myers RM, Sidow A: Genome-wide analysis of transcription factor binding sites based on ChIP-Seq data. Nat Methods 2008, 5:829-834.

16. Lee BK, Bhinge AA, Battenhouse A, McDaniell RM, Liu Z, Song L, Ni Y, Birney E, Lieb JD, Furey TS, Crawford GE, Iyer VR: Cell-type specific and combinatorial usage of diverse transcription factors revealed by genome-wide binding studies in multiple human cells. Genome Res 2012, 22:9-24.

17. ENCODE Project Consortium: The ENCODE (ENCyclopedia Of DNA Elements) Project. Science 2004, 306:636-640.

18. Whitington T, Frith MC, Johnson J, Bailey TL: Inferring transcription factor complexes from ChIP-seq data. Nucleic Acids Res 2011, 39(15):e98.

19. van Steensel B: Chromatin: constructing the big picture. EMBO J 2011, 30:1885-1895.

20. Moorman C, Sun LV, Wang J, de Wit E, Talhout W, Ward LD, Greil F, Lu XJ, White KP, Bussemaker HJ, van Steensel B: Hotspots of transcription factor colocal-ization in the genome of Drosophila melanogaster. Proc Natl Acad Sci USA 2006, 103:12027-12032.

21. Negre N, Brown CD, Ma L, Bristow CA, Miller SW, Wag-ner U, Kheradpour P, Eaton ML, Loriaux P, Sealfon R, Li Z, Ishii H, Spokony RF, Chen J, Hwang L, Cheng C, Auburn RP, Davis MB, Domanus M, Shah PK, Mor-rison CA, Zieba J, Suchy S, Senderowicz L, Victorsen A, Bild NA, Grundstad AJ, Hanley D, MacAlpine DM, Mannervik M, Venken K, Bellen H, White R, Gerstein M, Russell S, Grossman RL, Ren B, Posakony JW, Kellis M, White KP: A cisregulatory map of the Drosophila genome. Nature 2011, 471:527-531.

22. Ellrott $\mathrm{K}$, Yang C, Sladek FM, Jiang T: Identifying transcription factor binding sites through Markov chain optimization. Bioinformatics 2002, 18(Suppl 2):S100-109.

23. Duda K, Chi Yl, Shoelson SE: Structural basis for HNF-4alpha activation by ligand and coactivator binding. I Biol Chem 2004, 279:23311-23316.

24. Zhang X, Wrzeszczynska MH, Horvath CM, Darnell JE: Interacting regions in Stat3 and c-Jun that participate in cooperative transcriptional activation. Mol Cell Biol 1999, 19(10):7138-7146.

25. Ito $T$, Tanahashi H, Misumi Y, Sakaki Y: Nuclear factors interacting with an interleukin-6 responsive element of rat alpha 2-macroglobulin gene. Nucleic Acids Res 1989, 17(22):9425-9435.

26. Leu Jl, Crissey MA, Leu JP, Ciliberto G, Taub R: Interleukin-6-induced STAT3 and AP-1 amplify hepatocyte nuclear factor 1-mediated transactivation of hepatic genes, an adaptive response to liver injury. Mol Cell Biol 2001, 21(2):414-424.

27. Schaefer TS, Sanders LK, Nathans D: Cooperative transcriptional activity of Jun and Stat3 beta, a short form of Stat3. Proc Natl Acad Sci USA 1995, 92(20):9097-9101.

28. Schuringa JJ, Timmer H, Luttickhuizen D, Vellenga E, Kruijer W: c-Jun and cFos cooperate with STAT3 in IL-6-induced transactivation of the IL- 6 respone element (IRE). Cytokine 2001, 14(2):78-87.

29. Schaefer TS, Sanders LK, Nathans D: Cooperative transcriptional activity of Jun and Stat3 beta, a short form of Stat3. Proc Natl Acad Sci USA 1995, 92(20):9097-9101.

30. Vesely PW, Staber PB, Hoefler G, Kenner L: Translational regulation mechanisms of AP-1 proteins. Mutat Res 2009, 682:7-12.

31. Oliviero S, Robinson GS, Struhl K, Spiegelman BM: Yeast GCN4 as a probe for oncogenesis by AP-1 transcription factors: transcriptional activation through AP-1 sites is not sufficient for cellular transformation. Genes Dev 1992, 6(9):1799-1809.

32. Maclsaac KD, Wang T, Gordon DB, Gifford DK, Stormo GD, Fraenkel E: An improved map of conserved regulatory sites for Saccharomyces cerevisiae. BMC Bioinformatics 2006, 7:113. 
33. De Rijcke M, Seneca S, Punyammalee B, Glansdorff N, Crabeel M: Characterization of the DNA target site for the yeast ARGR regulatory complex, a se-quence able to mediate repression or induction by arginine. Mol Cell Biol 1992, 12:68-81.

34. Dubois $E$, Messenguy F: Isolation and characterization of the yeast ARGRII gene involved in regu-lating both anabolism and catabolism of arginine. Mol Gen Genet 1985, 198(2):283-289.

35. Reed BD, Charos AE, Szekely AM, Weissman SM, Snyder M: Genome-wide occupancy of SREBP1 and its partners NFY and SP1 reveals novel functional roles and combinatorial regulation of distinct classes of genes. PLOS Genet 2008, 4(7):e1000133.

36. van der Watt PJ, Leaner VD: The nuclear exporter, $\mathrm{Crm} 1$, is regulated by NFY and Sp1 in cancer cells and repressed by p53 in response to DNA damage. Biochim Biophys Acta 2011, 1809(7):316-326.

37. Dalvai M, Mondesert O, Bourdon JC, Ducommun B, Dozier C: Cdc25B is negatively regulated by $\mathrm{p} 53$ through $\mathrm{Sp} 1$ and NF-Y transcription factors. Oncogene 2011, 30(19):2282-2288.

38. Borestrom C, Zetterberg H, Liff K, Rymo L: Functional interaction of nuclear factor $y$ and $\mathrm{sp} 1$ is required for activation of the epstein-barr virus C pro-moter. J Virol 2003, 77(2):821-829.

39. Hayden MS, Ghosh S: NF-kB, the first quarter-century: remarkable progress and outstanding questions. Genes Dev 2012, 26:203-234.

40. Ruefli-Brasse AA, French DM, Dixit VM: Regulation of NF-kappaBdependent lymphocyte activation and development by paracaspase. Science 2003, 302(5650):1581-1584.

41. Kamiya M, Judson H, Okazaki Y, Kusakabe M, Mu-ramatsu M, Takada S, Takagi N, Arima T, Wake N, Kamimura K, Satomura K, Hermann R, Bonthron DT, Hayashizaki Y: The cell cycle control gene ZAC/PLAGL1 is imprinted-a strong candidate gene for transient neonatal diabetes. Hum Mol Genet 2000, 9(3):453-460.

42. Meyer N, Penn LZ: Reflecting on 25 years with MYC. Nat Rev Cancer 2008, 8(12):976-990

43. Liu PY, Hsieh TY, Liu ST, Chang YL, Lin WS, Wang WM, Huang SM: Zac1, an Sp1-like protein, regulates human p21(WAF1/Cip1) gene expression in HeLa cells. Exp Cell Res 2011, 317(20):2925-2937.

44. Jarmalaite S, Laurinaviciene A, Tverkuviene J, Kalin-auskaite N, Petroska D, Bohling T, Husgafvel-Pursiainen K: Tumor suppressor gene ZAC/PLAGL1: altered expression and loss of the nonimprinted allele in pheochromocytomas. Cancer Genet 2011, 204(7):398-404.

45. Euskirchen GM, Rozowsky JS, Wei CL, Lee WH, Zhang ZD, Hartman S, Emanuelsson O, Stolc V, Weissman S, Gerstein MB, Ruan Y, Snyder M: Mapping of transcription factor binding regions in mam-malian cells by ChIP: comparison of array- and sequencing-based technologies. Genome Res 2007, 17:898-909.

46. Hudson ME, Snyder M: High-throughput methods of regulatory element discovery. BioTechniques 2006, 41, 673, 675, 677 passim.

47. Pages H: BSgenome.Hsapiens.UCSC.hg19: Homo sapiens (Human) full genome (UCSC version hg19) 2011

48. Bryne JC, Valen E, Tang MH, Marstrand T, Winther O, da Piedade I, Krogh A, Lenhard B, Sandelin A: JASPAR, the open access database of transcription factor-binding profiles: new content and tools in the 2008 update. Nucleic Acids Res 2008, 36:D102-106

49. Newburger DE, Bulyk ML: UniPROBE: an online database of protein binding microarray data on protein-DNA interactions. Nucleic Acids Res 2009, 37:77-82

50. Hubbard T, Barker D, Birney E, Cameron G, Chen Y, Clark L, Cox T, Cuff J, Curwen V, Down T, Durbin R, Eyras E, Gilbert J, Hammond M, Huminiecki L, Kasprzyk A, Lehvaslaiho H, Lijnzaad P, Melsopp C, Mon-gin E, Pettett R, Pocock M, Potter S, Rust A, Schmidt E, Searle S, Slater G, Smith J, Spooner W, Stabenau A, Stalker J, Stupka E, Ureta-Vidal A, Vastrik I, Clamp M: The Ensembl genome database project. Nucleic Acids Res 2002, 30:38-41.

51. Jothi R, Cuddapah S, Barski A, Cui K, Zhao K: Genome-wide identification of in vivo protein-DNA binding sites from ChIP-Seq data. Nucleic Acids Res 2008, 36: 5221-5231.

52. Phillips JE, Corces VG: CTCF: master weaver of the genome. Cell 2009, 137:1194-1211

53. Kim TH, Abdullaev ZK, Smith AD, Ching KA, Louki-nov Dl, Green RD, Zhang MQ, Lobanenkov W, Ren B: Analysis of the vertebrate insulator protein CTCF-binding sites in the human genome. Cell 2007, 128:1231-1245.
54. Grant CE, Bailey TL, Noble WS: FIMO: scanning for occurrences of a given motif. Bioinformatics 2011, 27:1017-1018.

55. Gupta S, Stamatoyannopoulos JA, Bailey TL, Noble WS: Quantifying similarity between motifs. Genome Biol 2007, 8:R24.

doi:10.1186/1471-2164-14-S5-S2

Cite this article as: Goi et al:: Cell-type and transcription factor specific enrichment of transcriptional cofactor motifs in ENCODE ChIP-seq data. BMC Genomics 2013 14(Suppl 5):S2.

\section{Submit your next manuscript to BioMed Central and take full advantage of:}

- Convenient online submission

- Thorough peer review

- No space constraints or color figure charges

- Immediate publication on acceptance

- Inclusion in PubMed, CAS, Scopus and Google Scholar

- Research which is freely available for redistribution

Submit your manuscript at www.biomedcentral.com/submit
Ciomed Central 